\title{
Railway disruption management challenges and possible solution directions
}

\author{
Nadjla Ghaemi ${ }^{1}$ (D) Oded Cats $^{1} \cdot$ Rob M. P. Goverde $^{1}$
}

Accepted: 27 February 2017/Published online: 17 March 2017

(C) The Author(s) 2017. This article is an open access publication

\begin{abstract}
This paper investigates the challenges of railway traffic controllers in dealing with big disruptions and the kind of support tools that could help to improve their task in terms of performance, lead time and workload. The disruption handling process can be partitioned into three phases resembling a bathtub. For each phase the essential decision making process has been identified. Currently, the support to rail traffic controllers in case of severe disruptions is limited to predefined contingency plans that are not always feasible or applicable. In the literature, models and algorithms have been identified that could be used in the different parts of the three phases of the disruption handling process. This paper investigates the processes of disruption management in practice and the challenges that traffic controllers are facing during a disruption. The literature of models applicable to disruption management is reviewed and classified based on the three phases of the traffic state during disruptions. Finally, a rescheduling optimization model is applied to a case of complete blockage on a corridor of the Dutch railway network. The case study shows how a microscopic model could support the traffic controllers by providing real-time solutions for different phases of a disruption.
\end{abstract}

Keywords Railway disruption · Rescheduling · Optimization

Nadjla Ghaemi

N.Ghaemi@tudelft.nl

Oded Cats

O.Cats@tudelft.nl

Rob M. P. Goverde

R.M.P.Goverde@tudelft.nl

1 Department of Transport and Planning, Delft University of Technology, P.O. Box 5048, 2600 GA Delft, The Netherlands 


\section{Introduction}

In case of large disruptions (e.g. infrastructure failures, rolling stock breakdown, accidents, etc.) railway traffic controllers should apply fast and proper measures to resolve the train services and prevent delay propagation to the rest of the network. Currently, predefined solutions called contingency plans are used to assist traffic controllers in dealing with disrupted traffic in the Netherlands and in other countries like Germany, Switzerland, Denmark and Japan (Chu and Oetting 2013). Each contingency plan corresponds to a specific disruption scenario in a specific location designed manually by experienced traffic controllers. The disadvantages of these plans are that they are not worked out in detail on infrastructure allocation level and cannot cover all the disruption cases throughout the network. They are constantly getting designed and updated based on the changes in timetable and infrastructure. In practice, it might happen that no suitable contingency plan is available for a disruption case. For such cases the traffic controllers are faced with a high workload to reach an agreement about the suitable plan. Since these plans are static and inflexible, the traffic controllers need to make some adjustments before being able to implement them. Hence, an algorithm that computes a new timetable for both sides of the disruption area is needed in practice. We believe that a feasible solution requires formulating the operation and infrastructure with fine granularity. Thus, in our research a great importance is given to methods with a microscopic level of detail.

The traffic level during disruptions resembles a bathtub, as is shown in Fig. 1. This bathtub model is divided into three phases. When a disruption happens, the traffic will decrease (first phase). The traffic remains at a low level during the disruption where a disruption timetable is applied based on the contingency plans (second phase). When the disruption has been solved the traffic will be recovered to the original timetable (third phase). The first and third phases are called transition phases, since they represent transitions of the operations from the original timetable to the disruption timetable and vice versa. In transition phases the traffic is not as regular as the traffic in the second phase or in the undisturbed situation. Those services that are decided to be cancelled in the disruption timetable should be handled in the first transition phase. In the third phase the cancelled services need to resume their operations. One of the drawbacks of the contingency plans is related to the lack of any instruction on how to deal with the transition phases.

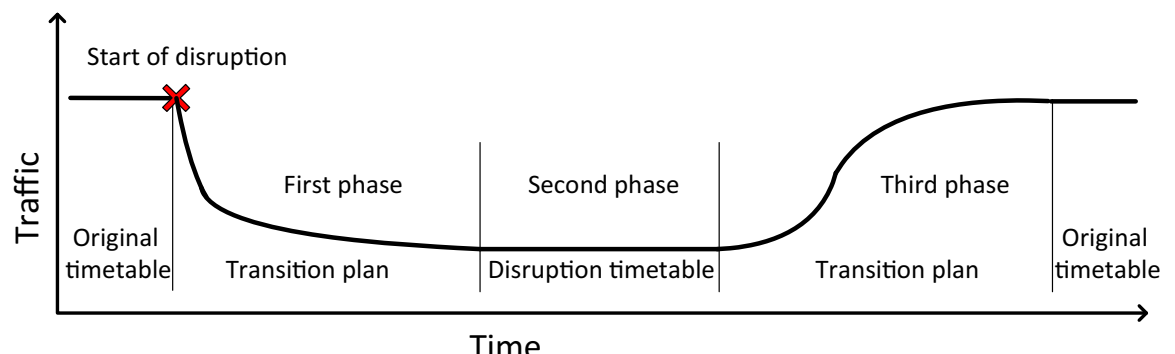

Fig. 1 Bathtub model illustrating the traffic levels during a disruption 
In this paper the problems that railway traffic controllers face when dealing with disruptions are investigated based on the Dutch practice. Then these problems are classified based on the three phases of the bathtub model. Next a critical review of the models and approaches known from the literature is carried out. In the following step the applicability of the models for the defined problems is investigated, and an illustrative case shows the applicability of a microscopic model to a case of a complete track blockage. The contributions of the paper are as follows:

- Identification of the challenges of traffic controllers in disruption management based on interviews with practitioners.

- Classification of the existing approaches in literature according to the bathtub model.

- Demonstration of the support provided by a microscopic rescheduling model in different phases of a disruption.

The structure of the paper is as follows. Section 2 describes the current disruption management practice from the Netherlands and identifies the problems that need improvements. Section 3 reviews relevant disruption management models from the literature. Section 4 provides an illustrative example on how a microscopic model could support the disruption management within the three phases. Conclusions are given in Sect. 6.

\section{Disruption management in practice}

This section describes the practice of disruption management focusing on the Netherlands, and identifies the problems encountered based on interviews with traffic controllers, contingency plan developers, and railway control staff.

\subsection{Design of contingency plans}

Contingency plans are designed by experts who used to be signallers or traffic controllers. The design of these pre-defined solutions is based on the basic hour patterns of the Dutch timetable and station track occupations. Based on these patterns and a specific disruption scenario, the planner estimates the remaining capacity and decides which trains should be cancelled or short-turned. The cancellation of services should be divided between the different railway undertakings that are operating in the area. Then the stations at which the trains should be short-turned are defined. For each corridor so-called decoupling stations are defined in advance where trains will short-turn in case of a complete blockage. Different train types (e.g. Intercities or local trains) may have different decoupling stations for short-turning. In defining the short-turning locations, it is anticipated that the shortturned trains replace the trains in the opposite direction. Based on the station track occupation, it is checked whether the trains could short-turn at the proposed time and platform. These static solutions are not able to consider the inherent 
uncertainties of the real-time operations and thus their realization might not be possible if the actual traffic deviates from the basic patterns.

\subsection{Workflow of disruption management}

Since 2010, the Netherlands has a centralized Operation Control Centre Rail (OCCR) to face large disruptions. The aim of having a centralized control centre is to bring different railway stakeholders such as the traffic controllers from the Infrastructure Manager (IM), the operations controllers from the Railway Undertakings (RU) and the delegates from the contractors together to achieve a higher performance by better communication. This becomes especially important with big disruptions on the network when the stakeholders have to cooperate closely. Figure 2 shows the workflow during a disruption. If the train traffic is hampered due to a serious failure of infrastructure or rolling stock, it is usually the driver who first notices the problem. This information is communicated to the back office (BO) of the OCCR through the decentralized control center by a signaller. Then, an inspector (a.k.a. general controller) is sent to the location of the failure to provide updates about the status of the problem to the back office. In the meantime the back office creates an announcement notification in the online traffic control information system, so the signallers, traffic controllers and other involved actors could access the announcement and get informed. In this notification, the problem, the people who should be involved, and the specific location that should be identified by the signaller are mentioned. The involved actors are able to modify and update the provided information online.

If required, contractors are sent to the location to repair the problem. Meanwhile the railway undertakings should deal with the disturbed trains that cannot proceed according to their original schedule. Based on the information from the field such as the location and severity of the disruption, the relevant contingency plan is selected and communicated with the traffic controllers from the infrastructure manager. Before implementing any plan, first it should be agreed between the traffic controllers of the infrastructure manager and the railway undertakings that the

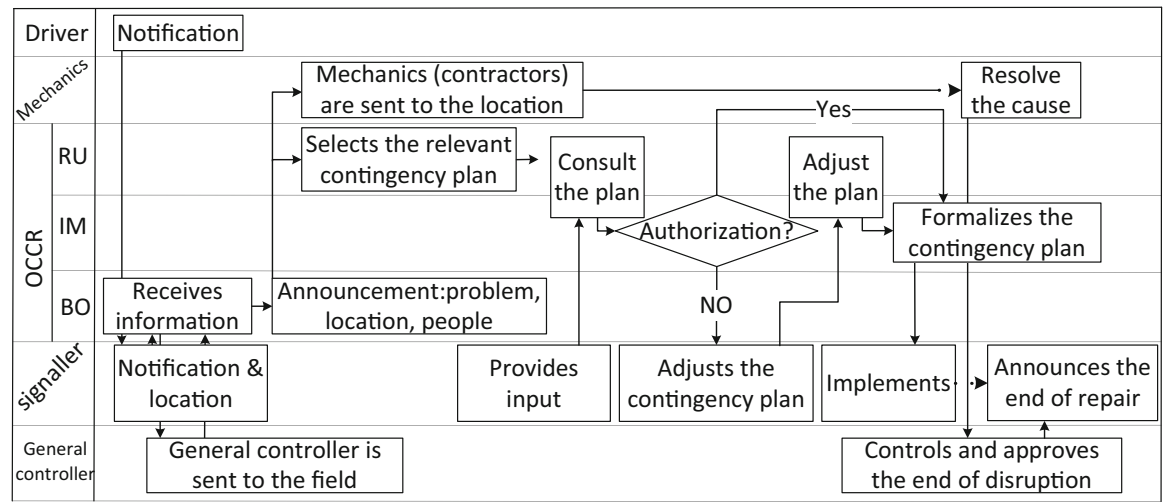

Fig. 2 Workflow of disruption management 
selected plan offers a suitable solution for the disrupted situation. Finally the traffic controllers in the OCCR should authorize the implementation of the contingency plan. In case the contingency plan requires some adjustments, this should be performed in consultation with the signallers who are responsible for route setting. Once authorized the contingency plan will be formalized by the traffic controllers and implemented by the signaller. After the repair crew solved the cause of the disruption and this has been approved by the general controller, the termination of the repair in the field will be announced in the online information system. The traffic can restore as soon as the disruption is over. However, as is shown in Fig. 1, the third phase may take some time for the transition from the disruption timetable to the original timetable.

\subsection{Identified problems in the OCCR}

In this section the difficulties regarding the processes mentioned earlier are presented and projected on the three phases of the bathtub model. The first phase starts as soon as the traffic becomes disturbed due to an unplanned event or when an incident is communicated to the back office. It takes some time before the precise location of the disruption is known and communicated. The situation is communicated to the back office of OCCR where a decision should be taken. The decision about implementing a contingency plan in the first place depends on the disruption length estimation. If the estimated length is less than $45 \mathrm{~min}$ then it is preferred not to implement any contingency plan. Thus, it is important to have a fast and accurate disruption length estimation which is currently missing. If it is expected that the disruption lasts longer than $45 \mathrm{~min}$, the search for a suitable contingency plan starts. This search is based on the information received from the field such as the exact location of the disruption and its severity.

In case of an existing suitable contingency plan, there is a problem regarding the implementation of these plans in the short-turning stations. The contingency plans correspond to the second phase of the bathtub model with the reduced traffic. However, since the detailed information regarding the implementation of this reduction depends on the real state of the traffic, the solution cannot be specified in the contingency plan exactly. It might happen that at the moment when the suitable contingency plan is selected, the train already left the station where it had to short-turn and the traffic controller needs to take care of the operation of this train in the following station. Thus, the traffic reduction might not be implemented as straightforward as is suggested in the contingency plan. Therefore, these plans do not provide sufficient detailed information about the processes that were unplanned in the original timetable. Since these contingency plans are predefined, they may need to get adjusted to reflect the real traffic status. For example, if the trains do not operate according to the plan and their platform track occupation does not correspond to the planned pattern, then the specific station platform might still be occupied by another train and accordingly a suggested short-turning might not take place at the defined time or platform.

Nevertheless, the most relevant contingency plan is chosen by the RU operations controllers and then modified to a disruption timetable in collaboration with the IM 
traffic controllers in the OCCR. A problem might occur when the traffic controller and signaller do not agree on a decision such as cancelling a service and have different opinions about which decision should be taken. Then reaching an agreement might take long and, moreover, the final decision might not be the optimum, since it depends on the experience of the traffic controller and signaller. If no suitable contingency plan is available then the traffic controllers are in charge of providing a feasible plan based on the actual traffic state. The common practice is to isolate the disrupted area and prevent delay propagation to other lines. The services that are directly affected by the disruption should be identified and handled separately. This task is rather difficult, especially in the main stations with many trains. In current practice, handling the disruption directly depends on the experience and skill of the person in charge. This is the main reason of disagreements between the controllers and signallers.

In the second phase any new information about the actual state of the disruption might require some adjustments to the current operation. In this phase, it is also important to plan ahead to restore the original timetable. Therefore, in this phase the information about the disruption length plays an important role. If accurate information about the disruption length is available, the third phase could be planned to achieve a smooth and fast transition from the disruption timetable back to the original timetable. In the third phase, it is important to reinsert the cancelled services and restore the original plan in such a way that it does not hamper the traffic of the adjacent areas. Table 1 summarizes the identified challenges in each phase. Looking at the identified problems, it can be concluded that the traffic control faces most problems during the first phase where the uncertainty regarding the exact disruption and a suitable solution is the highest.

\section{Literature study}

There is a rich literature and overview of models and methods used for dealing with operational uncertainties. However, there are limited references addressing the large disruptions where many trains should be cancelled or short-turned. Cacchiani et al.

Table 1 Identified challenges in each phase

\begin{tabular}{ll}
\hline Phase & Challenges identified \\
\hline First phase & Receiving precise information about the disruption location \\
& Estimating the disruption length \\
& Discussing the decision and adjusting the contingency plan \\
& Isolating the disrupted area (in case of no contingency plan) \\
& Adjusting and implementing the disruption timetable \\
Second phase & Estimating the remaining disruption length \\
& Preparing the transition phase \\
& Reinserting the cancelled services \\
Third phase & Restoring the original plan
\end{tabular}


(2014) provide an overview of models and algorithms for real-time rescheduling. In this overview, the literature is classified into two categories. The models and methods that are handling relatively small deviations from the scheduled timetable referred to as disturbances, and those which deal with large deviations that usually involve long delays and cancellation of services and rescheduling of rolling stock and crews which are referred to as disruptions. The models for disturbances and disruptions are developed based on either microscopic or macroscopic detail of the infrastructure and operations. The review concludes that the research on disruption management and especially with microscopic level of detail is surprisingly limited.

This section provides a review of the disruption literature with special attention to the three phases of the bathtub model. In this review the models are classified based on the number of different phases they are applicable to. It is also indicated whether they consider a micro or macro level of detail. Within this classification the applications of the approaches are also divided into those models that compute a new schedule and those that provide insight into any of the three phases. Note that in our review crew rescheduling is not included. The relevance of models to the different phases are determined based on the characteristics of each phase, which are as follows:

- First phase: disruption length uncertainty, service cancellation and its impact on the operating services.

- Second phase: disruption length uncertainty, disruption timetable.

- Third phase: service reinsertion and its impact on the operating services.

Section 3.1 reviews the relevant literature and classifies them to one or more of the phases. Then Sect. 3.2 presents the application of these approaches to dealing with the challenges mentioned in Sect. 2.3 per phase.

\subsection{The disruption models}

\subsubsection{Models dealing with one phase}

Despite the importance of short-turning strategies in case of disruptions, there are only limited references that investigated this topic. Coor (1997) macroscopically modelled a high-frequency single transit line to simulate short-turning trains with the objective to decrease the passenger waiting times. He concluded that a shortturning strategy is more beneficial in case of severe delays than small delays. The model provides insight about the advantages of short-turning for the second phase of the bathtub model.

Shen and Wilson (2001) developed a real-time disruption control model using mixed-integer linear programming (MILP). The macroscopic model considers a single line and formulates the route between stations as a sequence of block sections. Different control strategies such as short-turning, holding and stop skipping are tested. The authors conclude that the combination of holding and shortturning strategies reduces the mean passenger waiting time remarkably well. The 
model computes a schedule mainly for the second phase. Although the model could have been extended to the other phases this was not mentioned explicitly, so we disregard their relevance for the transition phases.

Jespersen-Groth et al. (2009) focus on the recovery transition from a disruption timetable to the original timetable. When a disruption occurs, the trains are shunted away to the closest depots in the same direction. After the cause of disruption has been resolved, first a train should take the train drivers from the central station to the depots so that the cancelled trains can resume their operations. This recovery is modelled macroscopically as a mixed integer program (MIP) to calculate the best reinsertion of cancelled services into the network to fit the periodic timetable.

Hirai et al. (2009) used Petri nets and integer programming (IP) to formalize and solve the train stop deployment problem. The model determines the stop locations for trains that can no longer operate according to the timetable and need to be cancelled. To avoid delay propagation, the focus is on isolating the disturbed area from other lines. The output of the model is a stop location for each train to clear the route for trains that are not disturbed and can still commute on other lines. The model is considered to be microscopic since the infrastructure is modelled at the level of block sections. This method partly addresses the first phase of the bathtub model with respect to the services that are cancelled due to a disruption but it does not provide any plan to the other trains or decides whether trains should be shunted or short-turned in case of a complete blockage.

Meng and Zhou (2011) used stochastic programing to incorporate the uncertainty of the disruption duration in probabilistic scenarios. The rescheduling is then performed based on a rolling horizon. The selected solution is the one with the minimum expected delay at the final station of all services. In this paper, the services resume as soon as the infrastructure is available, thus no other strategy such as short-turning or cancellations are considered and the focus is on the third phase.

Narayanaswami and Rangaraj (2013) developed an MILP for a single-track line. The only dispatching measure considered is delaying trains. The model assumes disruption length to be given, as well as the start and end time of the disruption. The decision variables of the model represent the arrival and departure of the trains in the station. The model is macroscopic and thus does not consider blocking times. Minimum process times and scheduled arrival and departure times are the inputs of the model. The objective is to minimize the weighted difference between the scheduled and actual arrival time at the final destination for all trains. The model computes the decision variables by delaying trains until the disruption is over and then defines the order and schedules of departing trains based on the weights. The disadvantage of this model is that the delay could propagate easily if the trains are not short-turned. The model is useful for the third phase of the bathtub model when the disruption cause is repaired and the operations can get back to the original timetable.

Chu and Oetting (2013) considered additional processes that are not planned but result from a disruption. The extra processes refer to communication, gathering information about the disruption, taking decisions about the suitable contingency plan and implementing the selected solution. To gain an insight about the first transition phase, they analysed the operational data of two big German urban 
railway networks where contingency plans were implemented. They concluded that one of the main reasons for delays during this phase was due to queuing of trains at the short-turning stations. Looking into the extra processes, they make a distinction between non-recurring and recurring processes. The first one refers to those specific processes that belong to specific trains (e.g. giving written orders train by train) which do not repeat and the second one refers to the ones that reoccur such as shortturnings. They highlighted the importance of these extra processes in deriving feasible contingency plans in stations using microscopic modelling of the blocking times. This research gives insight about the first phase of bathtub model.

The objective for managing large disruptions may be to maximize the service level. Louwerse and Huisman (2014) formulated the problem as a macroscopic MILP, considering both partial and complete blockages of a railway line. Their main focus is on computing the disruption timetable for the second phase. The original timetable and an estimation of the disruption duration are used as input of the model and the output of the model is the rescheduled timetable indicating which trains should run with their schedules.

\subsubsection{Models dealing with two phases}

Zhan et al. (2015) modeled a complete blockage by mixed integer linear programming. Their objective is to minimize total weighted delay and cancelled services considering headway and station capacity constraints. The output of the model is the decision about cancelled services, the stations where the affected trains need to wait until the disruption is over and the order of the departures. The model is developed for long distance services with seat reservations. In case of a disruption, the trains are not short-turned due to problems associated with rolling stock circulations. Since the model defines waiting locations for hindered services, it partly addresses the first phase. For the most part, the model deals with the third phase by computing the departure orders after the disruption cause is repaired.

Zilko et al. (2016) developed a model for estimating the disruption length. A Non-Parametric Bayesian Network (NPBN) is used to model the joint distribution between variables that characterizes the nature of the disruption. By conditioning on new information the estimation of the disruption length can be improved whenever information updates become available. Accurate estimates of the disruption length are very useful to achieve smooth transition phases. Thus, the model provides support for the first and second phases of the bathtub model.

\subsubsection{Models dealing with three phases}

Nakamura et al. (2011) developed a macroscopic model for dealing with a complete blockage on a double-track network. The model uses three predetermined factors: train group, train cancellation sections and short-turning patterns, which result in a train rescheduling pattern. The model cancels the services running in the disrupted area and connects the short-turning trains to the trains running in the opposite direction. Then it identifies those train lines that have either no assigned rolling stock or no planned route. At the final step of the algorithm, the process of matching 
the plans and rolling stock takes place. The main focus of the paper is to support the traffic controllers by proposing train cancellations and short-turnings. The support covers the three phases of the bathtub model.

Veelenturf et al. (2016) extend the macroscopic model of Louwerse and Huisman (2014). In the extended model, a real case of a railway network is used with more than two tracks between and inside stations, and the train services are able to use other tracks than they were originally assigned to. The objective of the model is to minimize delay and the number of cancelled services. The transition phases are implicitly addressed.

\subsection{Applicability of the models to the identified problems}

This section investigates the applicability of the reviewed models to the identified problems for each phase. Within each phase the applicable literature is sorted based on the order of the identified challenges in Table 1. Tables 2, 3 and 4 summarize the models over the three phases.

\subsubsection{First phase}

The traffic controllers face most challenges during the first phase. The first difficulty is to have an accurate estimation about the disruption length. Zilko et al. (2016) developed a model specifically for estimating the disruption length, which includes latency time and repair time. Knowing the approximate disruption length, the traffic controllers have to find the relevant contingency plan and implement it. Before implementing the contingency plan for the second phase, they also have to decide on a plan for the first phase which would eventually reach the disruption timetable suggested in the contingency plan. Thus, for the greatest extent, the plan for the first phase depends on the contingency plan of the second phase.

Chu and Oetting (2013) studied the effects of unplanned events that result in extended process times. The research provides a clear understanding on the capacity consumption in stations with short-turning; however, it does not provide a rescheduling model to compute a solution including short-turnings. The implementation of short-turning trains still needs to be investigated more at a microscopic level of detail.

Table 2 The identified challenges and relevant literature for the first phase

\begin{tabular}{ll}
\hline Problems & Models for the first phase \\
\hline Estimating disruption length & Zilko et al. (2016) \\
Identifying stop locations & Hirai et al. (2009) \\
Adjusting contingency plan, discussing decision & Zhan et al. (2015) \\
& Nakamura et al. (2011) \\
& Chu and Oetting (2013) \\
& Veelenturf et al. (2016)
\end{tabular}


Table 3 The identified challenges and relevant literature for the second phase

\begin{tabular}{ll}
\hline Problems & Models for the second phase \\
\hline Estimating remaining disruption length & Zilko et al. (2016) \\
Adjusting and implementing the disruption timetable & Coor (1997) \\
& Shen and Wilson (2001) \\
& Nakamura et al. (2011) \\
& Louwerse and Huisman (2014) \\
& Veelenturf et al. (2016) \\
\hline
\end{tabular}

Table 4 The identified challenges and relevant literature for the third phase

\begin{tabular}{ll}
\hline Problems & Models for the third phase \\
\hline Reinserting the cancelled services & Jespersen-Groth et al. (2009) \\
& Meng and Zhou (2011) \\
& Narayanaswami and Rangaraj (2013) \\
Zestoring the original plan & Zhan et al. (2015) \\
& Nakamura et al. (2011) \\
& Veelenturf et al. (2016) \\
\hline
\end{tabular}

Another problem in the first phase is how to adjust a contingency plan and reach an agreement on a decision. To address this problem, the papers on rescheduling that include cancelling and short-turning trains can be used. Nakamura et al. (2011), and Veelenturf et al. (2016) provide solutions for rescheduling that implicitly include the first phase. Nakamura et al. (2011) focus on avoiding delay propagation while Veelenturf et al. (2016) focus on minimizing delay and number of cancelled services. However, they do not provide microscopic insight into the station capacity consumption which is very important in the first phase.

The final problem concerns the cases where no contingency plan is available and traffic controllers should isolate the disrupted area to avoid delay propagation. Hirai et al. (2009) provide a model that can be used to calculate the stop positions for the trains that are affected directly so that the other trains could continue their trips conflict-free. The approach by Zhan et al. (2015) also defines the waiting location for trains until the source of the disruption is over. We believe that using a decision support tool can speed up the process of discussion and decision making.

\subsubsection{Second phase}

In the second phase the contingency plan might get adjusted with the updated information about the status of the disruption. For example, it might be the case that more (or fewer) routes should be cleared due to the disruption to access the tracks where the repair needs to be done. This results in the same problems of adjusting the plan and agreeing on a decision as in the first phase. Most literature available 
concerns the development of a disruption timetable for this phase such as Shen and Wilson (2001), Nakamura et al. (2011), Louwerse and Huisman (2014) and Veelenturf et al. (2016). Coor (1997) looked at short-turning trains as a strategy to compensate for the time loss in the second phase and the main conclusion confirms the benefits of short-turning trains in case of large disruptions. Also in the second phase a reliable estimation about the disruption length is required for which the model developed by Zilko et al. (2016) can be used.

\subsubsection{Third phase}

It is important to know when the disruption cause is expected to be resolved. This information is essential to plan for the third phase, where the train operations should switch from the disruption timetable to the original one. To give an example, if a reliable disruption length is available it can be decided earlier to stop the shortturning and operate trains based on the original plan again which shortens the second phase. The model of Meng and Zhou (2011) incorporates the uncertainty regarding this information and determines the order of trains to proceed after the disruption with the least delay. Narayanaswami and Rangaraj (2013) and Zhan et al. (2015) also contribute to the third phase, by computing the departure orders after the end of disruption. Jespersen-Groth et al. (2009) focus on resuming the operation taking into account the rolling stock circulation and crew. This model can also be used to develop a plan for reinserting the services for the third phase.

Nakamura et al. (2011) and Veelenturf et al. (2016) provide a plan for cancelled and short-turned trains, which helps the traffic controllers to know which cancelled and short-turned trains should be reinserted back in the network. However, the implementation of the plan in this phase requires a microscopic representation of the infrastructure and processes, especially in stations where trains were short-turned.

Table 5 gives a summary of the disruption models. The relevance of each model to each phase is shown by $\boldsymbol{}$. The column "Focus" indicates whether the model is rescheduling (R) or brings insight (I) to a particular phase. From the table we can conclude that there are limited disruption support models at a microscopic level of detail. There are two macroscopic models that address all three phases. However, the feasibility of these solutions should be checked with a microscopic model. Thus, a microscopic model that is able to address all three phases of a disruption is still missing in the literature. In the following section we will show how a microscopic model can provide support for the traffic controllers for each phase of a disruption.

\section{Application of a microscopic model to a disruption case}

This section illustrates the relevance and applicability of a microscopic approach for rerouting and rescheduling trains in the different phases of a disruption. Caimi et al. (2011) developed a resource-constrained multicommodity flow model originating from the node-packing approach by Zwaneveld et al. (1996) for rerouting and rescheduling, which can be applied to resources at a microscopic level of detail. The set-packing approach developed by Lusby et al. (2011) incorporates time and place 
Table 5 Summary of the reviewed disruption models

\begin{tabular}{|c|c|c|c|c|c|}
\hline Paper & Micro & Phase 1 & Phase 2 & Phase 3 & Focus \\
\hline Coor (1997) & - & - & $\boldsymbol{V}$ & - & I \\
\hline Shen and Wilson (2001) & - & - & レ & - & $\mathrm{R}$ \\
\hline Jespersen-Groth et al. (2009) & - & - & - & $\boldsymbol{r}$ & $\mathrm{R}$ \\
\hline Hirai et al. (2009) & $\boldsymbol{}$ & レ & - & - & $\mathrm{R}$ \\
\hline Nakamura et al. (2011) & - & レ & $\boldsymbol{V}$ & $\boldsymbol{\nu}$ & $\mathrm{R}$ \\
\hline Meng and Zhou (2011) & - & - & - & $\boldsymbol{r}$ & $\mathrm{R}$ \\
\hline Narayanaswami and Rangaraj (2013) & - & - & - & $\boldsymbol{r}$ & $\mathrm{R}$ \\
\hline Chu and Oetting (2013) & レ & レ & - & - & I \\
\hline Louwerse and Huisman (2014) & - & - & $\boldsymbol{V}$ & - & $\mathrm{R}$ \\
\hline Veelenturf et al. (2016) & - & $\boldsymbol{V}$ & $\boldsymbol{v}$ & レ & $\mathrm{R}$ \\
\hline Zhan et al. (2015) & - & レ & - & $\nu$ & $\mathrm{R}$ \\
\hline Zilko et al. (2016) & - & $v$ & 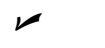 & - & I \\
\hline
\end{tabular}

dimensions to the problem formulation by considering each resource utilization in intervals of $15 \mathrm{~s}$. The disadvantage of time discretization is the possibility of missing any conflict that might take place between two discretized time points. In addition, both node-packing and set-packing approaches require pre-processing effort for computing resource utilization and conflict detection which eventually leads to limited rescheduling alternatives.

Pellegrini et al. (2014) proposed a Mixed Integer Linear Programing formulation for rescheduling and rerouting trains in complex junctions. The advantage of this formulation is that there is no need for pre-processing of the resource utilization to detect conflicts. Thus this formulation offers more scheduling alternatives. In this approach the conflicts are avoided by computing an order variable that prevents simultaneous resource utilization. Our rerouting approach is based on the model developed by Pellegrini et al. (2014) with the focus on short-turning services. The model computes the blocking time (Pachl 2014) of each track section used by any running train and finds a conflict-free route for each train while minimizing the total delay of all trains along their routes. The model is implemented in Matlab 2016a using YALMIP (Löfberg 2012) which is a free toolbox for fast implementation of optimization problems. Gurobi 2013 is used as solver on a laptop with an Intel(R) Core(TM) processor with $3 \mathrm{GHz}$ and $8 \mathrm{~GB}$ RAM. The computation time for all the cases (21 trains operating between 5 stations on 100 track sections) was less than $3 \mathrm{~s}$.

The aim of this case study is to illustrate the possible support that could be provided by a microscopic model to a traffic controller to manage the disruption during the different phases. For this case a railway corridor in the south of The Netherlands is selected. Figure 3 shows the corridor from station Nijmegen through Nijmegen Dukenburg (Nmd), Wychen (Wc), Ravenstein (Rvs), Oss (O) and further towards Den Bosch (Ht). This corridor is for the most part double-track, except between stations Wychen and Ravenstein where there is a single track (bridge) serving trains in both directions. The disruption occurs between station Oss and Den 
Bosch, thus all the arriving trains from Nijmegen have to short-turn in station Oss back to Nijmegen.

In the original timetable, two train lines operate between stations Nijmegen and Den Bosch: an intercity (IC) and a local train line (called sprinters (SP) in Dutch). To make a distinction between opposite services of the same line odd and even numbers are used depending on the travel direction. For example the services of the lines IC3600 and SP4400 run from station Den Bosch to Nijmegen on one track, and services of the lines IC3601 and SP4401 run in the opposite direction on the other track. The last two digits of the train line numbers indicate the operation time of that line during the day. For instance IC3617 departs at 06:18:00 from Nijmegen. The next IC train in the same direction departs half an hour later at 06:48:00 as IC3619. Both lines IC3600 and SP4400 operate with a frequency of two services per hour in each direction.

Due to the obstruction, trains coming from Nijmegen heading towards Den Bosch should be short-turned in station Oss and continue running back towards station Nijmegen. This short-turning implies a changed station track utilization with adjusted routes and platform track allocations that need to be checked for conflicts, acceptable track occupation and fit in the new timetable with preferably all shortturned trains running according to the original opposite scheduled train paths. Note that the running times and blocking times change due to the changed routes. Likewise, the platform track occupation time of a short-turning train also takes longer than the minimum dwell time for a continuing train.

Figure 4 shows the track layout in station Oss. In the original timetable, both SP and IC services run on the upper track from Nijmegen to Oss and on the lower track from Oss to Nijmegen. As can be seen the trains are able to use both platform tracks for short-turning in station Oss.

Table 6 shows the hourly pattern of the original timetable for the two train lines SP4400 and IC3600. The actual train numbers are represented by ** as they vary each hour. The departures and arrivals are indicated by the minutes in the hour. For instance the first row can represent the train IC3617 that departs from Nm at 06:18 and arrives in $\mathrm{O}$ at 06:32. The microscopic rescheduling model developed by Pellegrini et al. (2014) is used to compute the blocking time diagram for the original timetable of this corridor. Figure 5 plots the computed blocking time diagram of the services for the route operated by train line IC3600. The lack of visual blocks for the train line SP4400 in Nm is due to the fact that their departure platforms are different from those of line IC3600.

The planned timetable is shown by red dash-dotted lines for IC3600 services and red dotted lines for SP4400. The computed rescheduled trajectories are shown by solid blue lines passing through the blocks. To distinguish between the IC3600

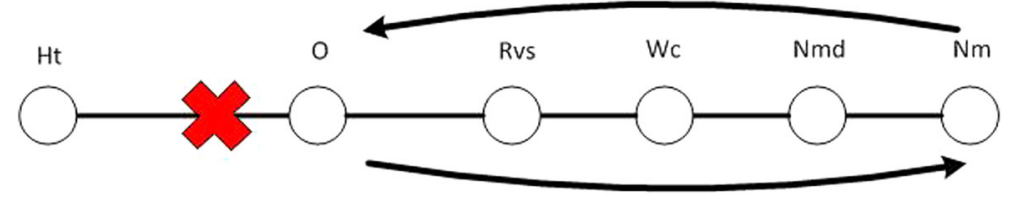

Fig. 3 An example of a complete blockage on a Dutch railway corridor 


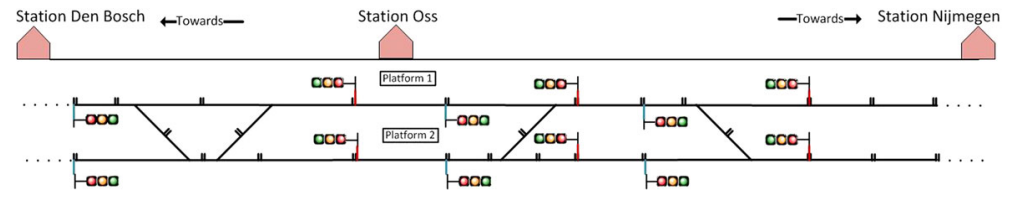

Fig. 4 The layout of station Oss where trains have to be short-turned

Table 6 Original timetable

\begin{tabular}{lll}
\hline Train lines & Dep from Nm & Arr to O \\
\hline IC36** & 18 & 32 \\
SP44** & 23 & 43 \\
IC36** & 48 & 02 \\
SP44** & 53 & 13 \\
Train lines & Dep from O & Arr to Nm \\
\hline SP44** & 14 & 35 \\
IC36** & 26 & 44 \\
SP44** & 44 & 05 \\
IC36** & 56 & 14 \\
\hline
\end{tabular}

services from SP4400 services, the planned departures and arrivals of the IC3600 services are marked as stars. In addition, the blocks of train line IC3600 are colored in magenta and the blocks of train line SP4400 are colored in cyan.

As mentioned before, the corridor is double track except between stations $\mathrm{Wc}$ and Rvs. Hence, the trains from station $\mathrm{O}$ to $\mathrm{Nm}$ run on another track than those running from $\mathrm{Nm}$ to $\mathrm{O}$ (besides the mutual single-track part). Thus, the blocking stairways from $\mathrm{O}$ to $\mathrm{Nm}$ shown in Fig. 5 are related to the single track between stations Rvs and Wc. Since all the services in both directions use the single track, the changes of the timetable including the order of services within the three phases are best understood by the single track blocking times at this location. Thus the blocking times of the single tracks are shown with different rectangles to emphasise the difference between the three phases of the disruption. The order of the operations of the train lines IC3600 and SP4400 on the single track between Wc and Rvs are shown with a solid rectangle in Fig. 5. This order represents the scheduled order of the original timetable. Two cases are defined to show how the optimal solution can be different given different disruption periods. Thus two cases are defined with a different start time of the disruption. The disruption in case 1 starts at 6:00 $\mathrm{AM}$ and in case 2 it starts at 6:30 AM. In both cases the disruption is over by 8:00 AM.

\subsection{Case 1: disruption starting at 6:00}

In this case the disruption period is assumed to be between 6:00 and 8:00. In the disruption time window, there are seven services (SP4417, IC3617, SP4419, 


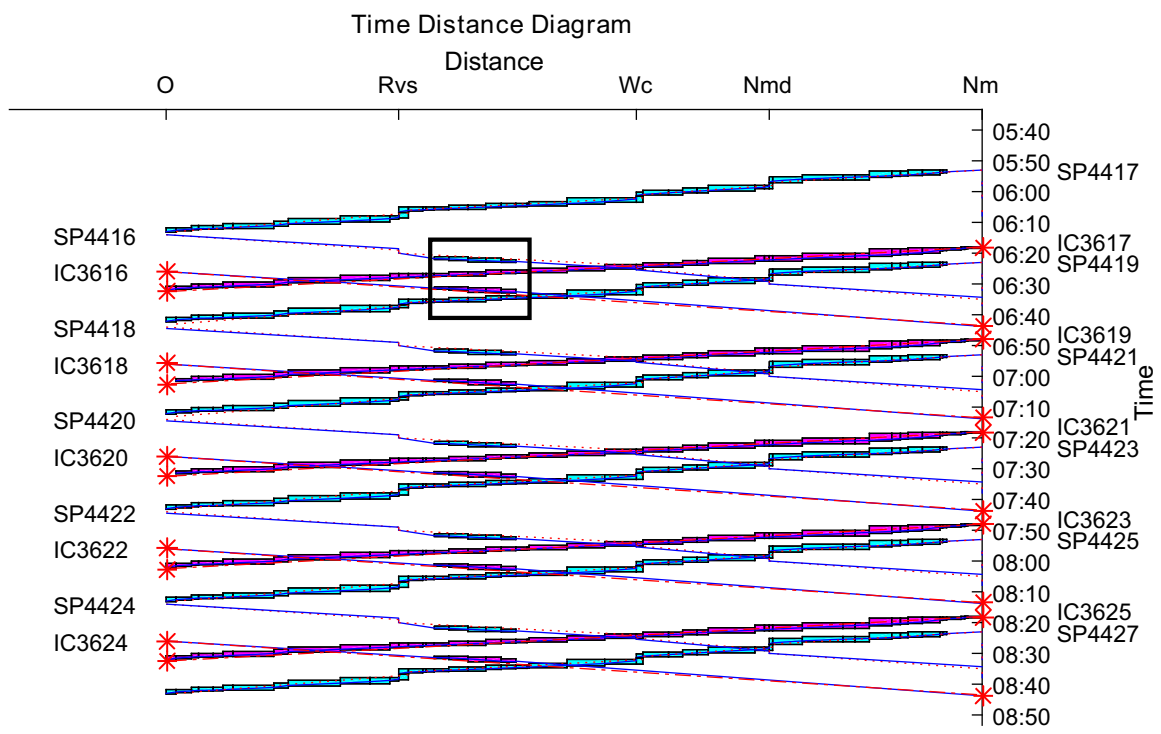

Fig. 5 Blocking time diagram of the original timetable

IC3619, SP4421, IC3621, SP4423) running from station Nm towards station O that arrive before 8:00. IC3623 is the first service that arrives at station $\mathrm{O}$ after 8:00 and is allowed to start using the restored section, after which also the services IC3623, SP4425, IC3625 and SP4427 resume their original operation. In the opposite direction, there are eight services (SP4416, IC3616, SP4418, IC3618, SP4420, IC3620, SP4422, IC3622) scheduled to operate from station $\mathrm{O}$ to $\mathrm{Nm}$ in the disruption period. Thus, in this particular case, seven arriving trains short-turn and can replace at most seven services from station $\mathrm{O}$ to $\mathrm{Nm}$. This would mean one service from station $\mathrm{O}$ to $\mathrm{Nm}$ needs to be cancelled. The choice of cancelling a service needs to be made by the traffic controllers, and the existing contingency plans do not provide any support in similar cases. Since the microscopic rescheduling model by Pellegrini et al. (2014) does not include the possibility of service cancellation, we have to predefine the cancelled service. Thus, two variants of Case 1 are considered with the assumption of cancelling the first IC service (IC3616) in the first variant and cancelling the last IC service (IC3622) in the second variant. The resulting computed blocking time diagrams are shown in Figs. 6 and 7.

Note that the services arriving from $\mathrm{Nm}$ to $\mathrm{O}$, use the upper platform track as shown in Fig. 4. Both time-distance diagrams shown in Figs. 6 and 7 plot the blocking times for the route starting from the IC platform track in Nm until the planned platform track used for the services arriving from Nm (upper track shown in Fig. 4). As shown in both figures, the optimal solution proposes that the services from line SP4400 short-turn on the upper track in station O. These short-turnings are shown by the cyan blocks in station O. Since the services of line IC3600 short-turn on the lower track these short-turnings are not shown in the Figs. 6 and 7 as the plotted blocks are related to the upper track. The computed platform choice is 


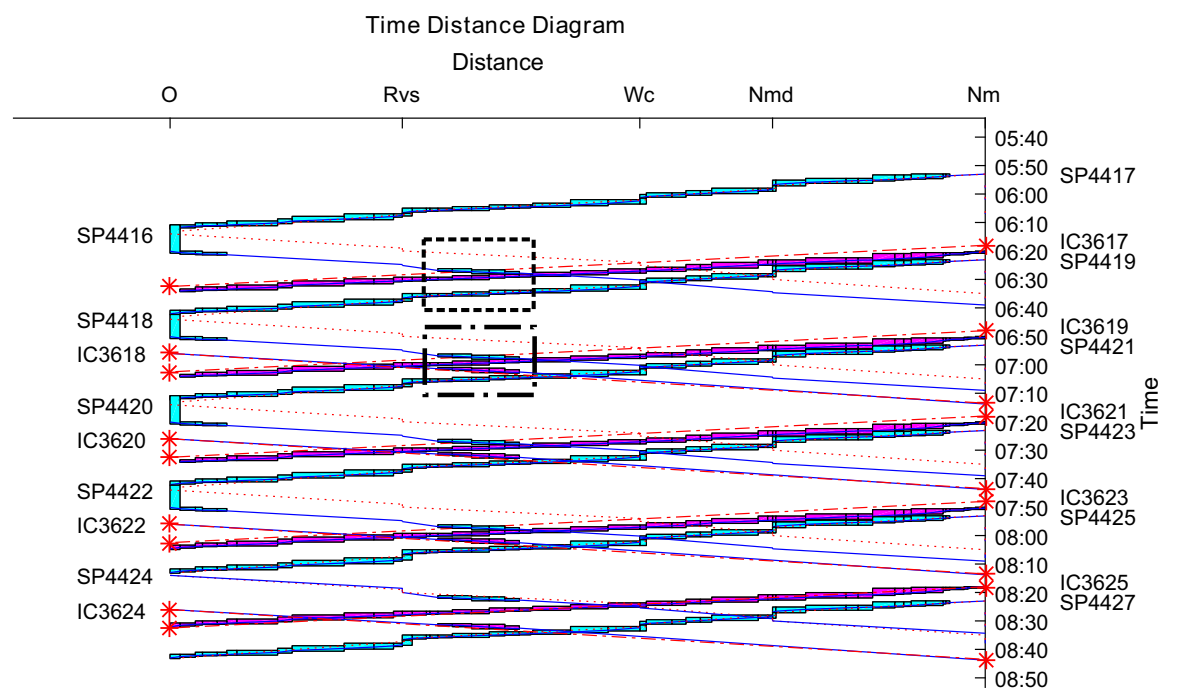

Fig. 6 Blocking time diagram when the disruption starts at 6:00 with IC3616 cancelled

consistent with the existing contingency plan. Since the rescheduling is performed for the whole period, all three phases of disruption are included in the result. The differences in the phases are easiest detected by checking the blocking time diagram of the single track between stations Wc and Rvs.

Figure 6 shows the first variant where service IC3616 has been cancelled. In this variant, every train that arrives at station $\mathrm{O}$, short-turns as the next service departing from $\mathrm{O}$. The SP services arrive $1 \mathrm{~min}$ before the original departures of the planned services towards $\mathrm{Nm}$.

However, we assume a minimum short-turning time of $8 \mathrm{~min}$. Thus, it is observed that the SP services are departing with 7 min delay. The delay is visualized by the difference between the dotted red line (the planned train paths of $\mathrm{SP}$ services from $\mathrm{O}$ to $\mathrm{Nm}$ ) and the solid blue lines (the computed train paths). The delay of SP services from $\mathrm{O}$ towards $\mathrm{Nm}$, introduce some delay to the IC services from $\mathrm{Nm}$ to $\mathrm{O}$. The reason for this delay is that in our formulation the delay penalties for SP and IC services are the same. From the optimization perspective, delaying an IC service that has one departure and one arrival is more favorable than delaying the SP service that has several stops in between. However, the choice of penalty can be easily changed based on the importance of the different services. The IC services arriving from $\mathrm{Nm}$ to $\mathrm{O}$ have enough time for short-turning, thus the departure delay from $\mathrm{Nm}$ does not propagate after the short-turning.

In Fig. 6 the first phase is shown with a dashed rectangle, where the IC3616 has been cancelled. The second phase of the disruption with a stable and repetitive pattern is shown in the dash-dotted rectangle. In this variant, the order of operation on the single track remained the same as in the original timetable with the blocking times being closer to each other. Thus, there is a smooth transition from the second 


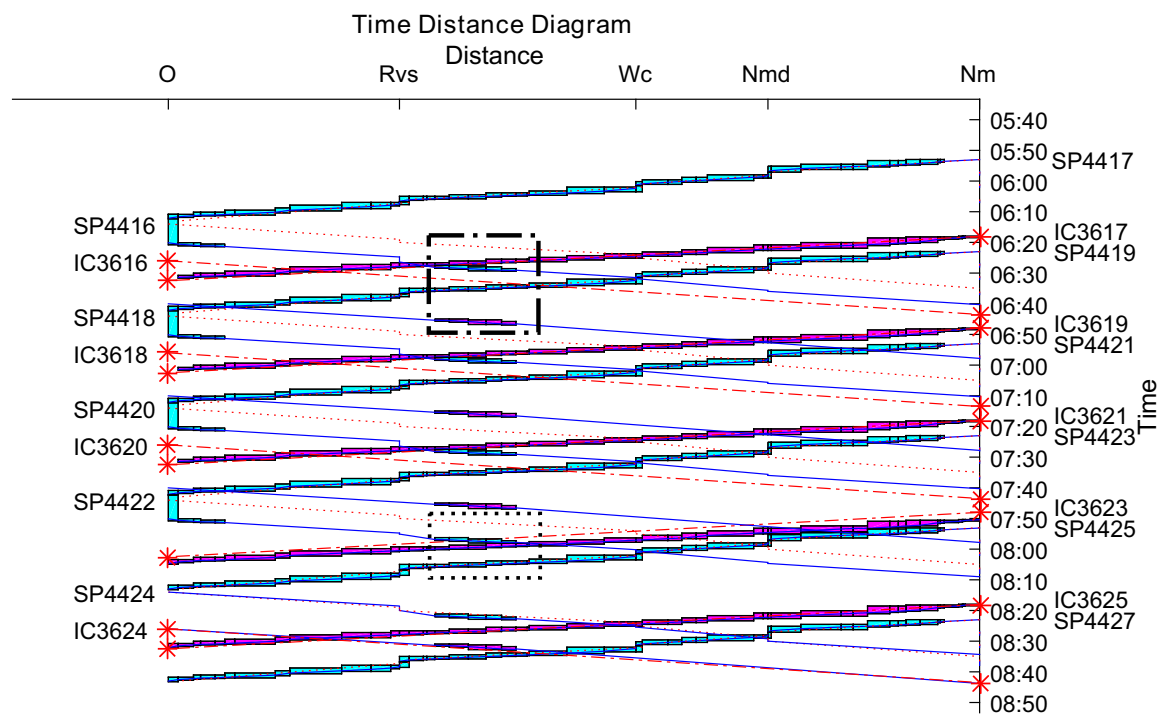

Fig. 7 Blocking time diagram when the disruption starts at 6:00 with IC3622 cancelled

phase to the original timetable as shown by services SP4424, IC3625, IC3624, SP4427.

Figure 7 shows the second variant where IC3622 has been cancelled. In this variant, the SP services from $\mathrm{Nm}$ to $\mathrm{O}$ short-turn as the next service departing from O. Similar to the previous variant, the short-turned service has $7 \mathrm{~min}$ of delay. The difference of this variant from the previous one is the choice of short-turning for the IC services. In this variant, the IC services from $\mathrm{Nm}$ to $\mathrm{O}$ replace the services from $\mathrm{O}$ to $\mathrm{Nm}$ that are scheduled to depart before their arrival. Thus, there is an unavoidable departure delay. In addition, the minimum short-turning time increases the departure delay of IC services from $\mathrm{O}$ to $\mathrm{Nm}$. In this variant, there is a smooth transition to the second phase. The dash-dotted rectangle shows the order of services operating on the single track in the second phase. In this variant, it is observed that the delay of SP4416 from O to Nm did not introduce any delay to the IC3617 from $\mathrm{Nm}$ to O. Instead, the SP4416 has more delay in comparison to the previous variant. This is due to the fact that, in case IC3617 is delayed, the delay would propagate through the short-turning. In this variant, the order of the operation is changed in the third phase. This is shown by a dotted rectangle. IC 3623 is the first service that starts using the blocked section after $\mathrm{O}$ and does not need to short-turn. Thus, there would be no short-turning delay propagation. However, it introduces a delay from $\mathrm{O}$ onwards which is not included in the model.

In the first variant the total arrival delay is $101 \mathrm{~min}$ and in the second variant it is $333 \mathrm{~min}$. The difference is due to the choice of short-turnings and the cancelled service. Hence, it is of great importance to compute the optimal choice of shortturning and cancelled services in case of disruption. Note that computing an optimal timetable for different phases of the disruption is not possible without having a reliable disruption length estimation. 


\subsection{Case 2: disruption starting at 6:30}

In this case, the disruption starts at 6:30 and ends at 8:00. Within this period, there are six services running from Nm to O (IC3617, SP4419, IC3619, SP4421, IC3621, SP4423). Note that SP4417 arrives at O before 6:30 and continues its original route. The same holds for the services in the opposite direction SP4416 and IC3616. So the services SP4418, IC3618, SP4420, IC3620, SP4422 and IC3622 need to be performed by the arriving trains to $\mathrm{O}$. In this case, there are six trains arriving and six scheduled services departing from $O$. Thus, there is no need for service cancellation. As mentioned earlier, the existing contingency plan suggests that the IC services short-turn on the lower track and the SP services short-turn on the upper track. In the original timetable without disruption both IC and SP services use the upper track to pass through station $\mathrm{O}$. It is probable that the disruption starts when the IC service is already on the upper track although the optimal solution proposes the lower track.

Figure 8 shows the optimal timetable in case the disruption period is from 6:30 to 8:00. In this case, the service IC3617 is already at the upper track when the disruption starts. So it needs to short-turn on the upper track in O. This would result in a different order of operation on the single track in the first phase which is shown by the dashed rectangle. The next IC services short-turn on the lower track. The resulting blocking time diagram on the single track for the next services are shown in the dash-dotted rectangle representing the second phase. Since the choice of short-turning in this case is the same as the first variant of the first case, the single track blocking time diagram in the second phase are the same for both cases. Similarly there is a smooth transition from the second phase to the original timetable starting from SP4424, IC3625, IC3624 and SP4427.

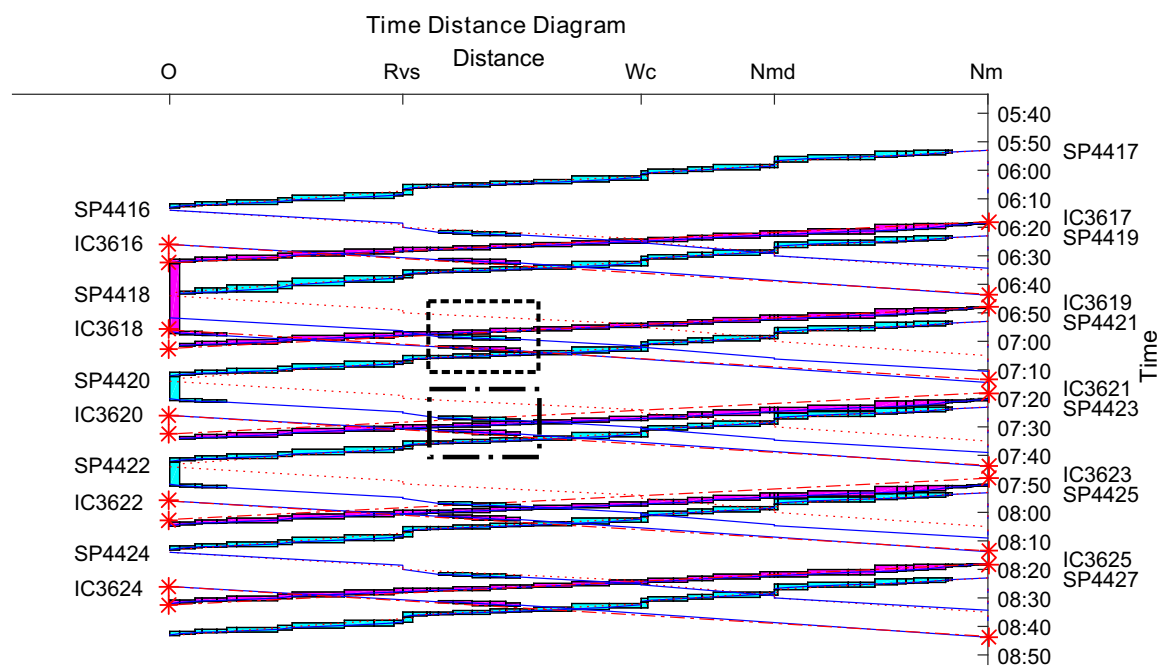

Fig. 8 Blocking time diagram when the disruption starts at 6:30 with IC3617 short-turning on the upper track 


\section{Discussion}

Depending on the disruption period there are different services affected. In the first case with a disruption from 6:00 to 8:00 there are seven arriving services and eight train services in the opposite direction. Thus, one service in the opposite direction should be cancelled. Since the existing contingency plans do not take into account the disruption period, they cannot provide any support regarding the short-turning choices and cancelled services. Different choices of short-turnings and cancelled services result in different timetables. The differences can be observed by the output of the microscopic rescheduling model for the two variants of the first case. With a microscopic model we are able to compute the blocking times of each track section and determine the optimal platform tracks for short-turnings. This cannot be done unless by taking into account the microscopic infrastructure and operational data. Moreover, the microscopic model provides insight about the order changes of services on the single track within the three phases. In the second case the disruption period is from 6:30 to 8:00. In this case there are six arriving services that would replace the six services in the opposite direction. The first IC service that should short-turn is already on the upper platform track when the disruption starts. However the computed optimal solution as well as the contingency plan suggest the lower platform track for the short-turning of IC services. This case shows that a static contingency plan cannot take into account such dynamic conditions and the suggested solution would not be feasible particularly in the transition phases.

\section{Conclusion}

In this paper the processes of disruption management and its relevant challenges were investigated, and the limitations of the current static contingency plans were discussed. The disruption models and algorithms in the literature were reviewed and classified based on the three phases of disruption. The literature study revealed that rerouting and rescheduling services during the transition phases are not sufficiently investigated. It was also concluded that limited microscopic models have been developed to deal with disrupted services. In the search for microscopic approaches, some relevant rescheduling models for small delays were reviewed. To illustrate the applicability of such methods for the three phases of disruption, a microscopic rescheduling model has been applied on a Dutch railway corridor. Since the microscopic rescheduling model was developed for traffic management of smaller delays and does not include the option of cancelling and short-turning train services, some assumptions were considered before applying the model to a disruption case. Two cases of disruptions were defined with different start times of a full blockage. It is concluded that depending on the disruption period, some services might need to be cancelled and different choices of short-turnings can be achieved. The results illustrated the support provided by a microscopic rescheduling model to traffic controllers for each phase of a disruption. Currently there is no reference in the literature and no support for the traffic controllers to decide which service is better 
to be cancelled and which choice of short-turnings results in the least total delay. The extension of a microscopic rescheduling model with cancellation and shortturning decisions will be a next research direction. Moreover, it is also interesting to investigate the possibility of short-turning the services in other stations.

Acknowledgements This research has been funded by the partnership programme ExploRail of technology foundation STW and the Dutch railway infrastructure manager ProRail in the project SmartOCCR under grant number 12257. The authors thank the interviewees from ProRail for sharing their knowledge about disruption management.

Open Access This article is distributed under the terms of the Creative Commons Attribution 4.0 International License (http://creativecommons.org/licenses/by/4.0/), which permits unrestricted use, distribution, and reproduction in any medium, provided you give appropriate credit to the original author(s) and the source, provide a link to the Creative Commons license, and indicate if changes were made.

\section{References}

Cacchiani V, Huisman D, Kidd M, Kroon L, Toth P, Veelenturf L, Wagenaar J (2014) An overview of recovery models and algorithms for real-time railway rescheduling. Transp Res Part B Methodol 63:15-37

Caimi G, Chudak F, Fuchsberger M, Laumanns M, Zenklusen R (2011) A new resource-constrained multicommodity flow model for conflict-free train routing and scheduling. Transp Sci 45(2):212-227

Chu F, Oetting A (2013) Modeling capacity consumption considering disruption program characteristics and the transition phase to steady operations during disruptions. J Rail Transp Plan Manag 3:54-67

Coor GT (1997) Analysis of the short-turning strategy on high-frequency transit lines by. Tech. rep. Massachusetts Institute of Technology, Massachusetts

Hirai C, Kunimatsu T, Tomii N, Kondu S, Takaba M (2009) A train stop deployment planning algorithm using a petri-net-based modelling approach. Q Rep RTRI 50(1):8-13. doi:10.2219/rtriqr.50.8

Jespersen-Groth J, Potthoff D, Clausen J, Huisman D, Kroon L (2009) Disruption management in passenger railway transportation. In: Robust and online large-scale optimization. Lecture notes in computer science, vol 5868. pp 399-421

Löfberg J (2012) Automatic robust convex programming. Optim Methods Softw 27(1):115-129

Louwerse I, Huisman D (2014) Adjusting a railway timetable in case of partial or complete blockades. Eur J Oper Res 235(3):583-593

Lusby R, Larsen J, Ryan D, Ehrgott M (2011) Routing trains through railway junctions: a new setpacking approach. Transp Sci 45(2):228-245

Meng L, Zhou X (2011) Robust single-track train dispatching model under a dynamic and stochastic environment: A scenario-based rolling horizon solution approach. Transp Res Part B Methodol 45(7):1080-1102

Nakamura T, Hirai C, Nishioka Y (2011) A practical train rescheduling algorithm using three predetermined factors. Tech. rep., presented at Proc. the 4th International Seminar on Railway Operations Modelling and Analysis (RailRome 2011), Rome, Italy, 2011

Narayanaswami S, Rangaraj N (2013) Modelling disruptions and resolving conflicts optimally in a railway schedule. Comput Ind Eng 64(1):469-481

Pachl J (2014) Timetable design principles. In: Pachl J, Hansen I (eds) Railway timetabling and operations, chap 2. Eurailpress, Hamburg, pp 23-24

Pellegrini P, Marlière G, Rodriguez J (2014) Optimal train routing and scheduling for managing traffic perturbations in complex junctions. Transp Res Part B Methodol 59:58-80

Shen S, Wilson NH (2001) An optimal integrated real-time disruption control model for rail transit systems. Computer-aided scheduling of public transport. Springer, Berlin, pp 335-363 
Veelenturf LP, Kidd MP, Cacchiani V, Kroon LG, Toth P (2016) A railway timetable rescheduling approach for handling large-scale disruptions. Transp Sci 50(3):841-862

Zhan S, Kroon LG, Veelenturf LP, Wagenaar JC (2015) Real-time high-speed train rescheduling in case of a complete blockage. Transp Res Part B Methodol 78:182-201

Zilko AA, Kurowicka D, Goverde RMP (2016) Modeling railway disruption lengths with copula bayesian networks. Transp Res Part C Emerg Technol 68:350-368

Zwaneveld PJ, Kroon LG, Van Hoesel SPM, Ambergen HW (1996) Routing Trains through railway stations: model formulation and algorithms. Transp Sci 128:14-33 